\title{
Practical Implementation of a Narrowband High Frequency Distributed Model for Locating Partial Discharge in a Power Transformer
}

\author{
S.D. Mitchell, J.S. Welsh, R.H. Middleton \\ School of Electrical Engineering and Computer Science, University of Newcastle, NSW Australia \\ B.T Phung \\ School of Electrical Engineering \& Telecommunications, University of NSW, NSW Australia
}

\begin{abstract}
This paper proposes the use of a narrowband high frequency based distributed transformer model for estimating partial discharge location within the winding of an interleaved power transformer. At high frequencies, the residual inductance within a transformer winding becomes significant. This inductive interaction generates its own characteristic response which, due to its distributed nature, can be utilized for partial discharge location. The technique proposed here takes advantage of the high frequency nature of a partial discharge and requires measurement at one location only. It also does not require detailed knowledge of the transformer's physical and material specifications. To confirm this approach, practical tests were conducted on a $66 \mathrm{kV} / 25 \mathrm{MVA}$ interleaved transformer winding. The partial discharge injection was delivered by an oil immersed point-plane $7.5 \mathrm{kV}$ source with the data captured using a current probe on the earth terminated neutral.
\end{abstract}

\section{INTRODUCTION}

A Partial Discharge (PD) as its name implies, is the partial breakdown of an insulation barrier within a system and a resulting exchange of charge. Partial discharges over a period of time, are not only the cause of insulation damage, which could ultimately lead to catastrophic failure, but are also useful in condition monitoring of insulation health [1]. The measured magnitude of a PD is an indicator of the severity of the fault.

Commercial partial discharge monitoring tools typically measure the magnitude (in picocoulombs), frequency of occurrence, and the relative phase position at which they occur. However these measurements can be misleading due to the fact that electrically they can only be practically observed outside the winding of the power transformer under study. The level of attenuation that the partial discharge undergoes during its passage through the winding can vary widely due to its dependence upon the location of the source of the PD. Therefore, an estimate of the location of the source based on an accurate transformer model would be a very useful tool for not only more accurate PD magnitude estimates, but also for transformer maintenance and repair [1-3].

Using the premise that a PD can be considered as an ideal current impulse, the detected PD's frequency response, coupled with an ac arate distributed model, can lead to the estimation of the PD site of origin [4]. In practice however, the recorded PD current signal is altered from a true impulse, with a quite noisy and colored spectrum.

\section{A. The Transformer Model}

\section{THEORY}

Partial Discharge localization techniques generally utilize a distributed model approach often approximated by a high order lumped parameter model. A simple RLC ladder network or distributed capacitor network are commonly used with quite good results $[5,6]$. However, these models are only suitable up to a few hundred $\mathrm{kHz}$ [7] and as such, fail to take advantage of the high frequency nature of a PD. Beyond these frequencies, traveling wave effects and the influence of the measurement equipment need to be considered [7].

To date there have been two fundamental approaches to modeling beyond a few hundred $\mathrm{kHz}$. The first is to ensure that no circuit element within the lumped parameter model has physically representational dimensions greater than one tenth of a wavelength of the frequencies being modeled [8, 9]. This will ensure that traveling wave effects up to the maximum modeling frequency are negligible. To accomplish this, the approach of most authors is to model a section for each turn [7]. This results in a prohibitively large yet still imperfect mathematical model [10]. The second approach, which is an area of active research, particularly in the transient overvoltage area, is the Multiconductor Transmission Line model (MLTL). MLTL treats the entry of a winding junction as multiple parallel transmission lines where an incident transient will be coupled into all paths [11].

Transmission line theory is still reliant upon a physically accurate model and some comparisons with the lumped parameter modeling approach have been found to be less accurate [12]. Akbari et al [13] describes the search for a high frequency model as an unsolved problem due to the nonlinear, frequency dependent nature of such a complex system. This paper sets out to provide a relatively simple high frequency model for the purpose of PD location by targeting a specific high frequency resonance and modeling in a narrow frequency band around this feature.

Due to its relatively low value of $\alpha$ (relationship between distributed series and shunt winding capacitance), an interleaved transformer winding facilitates the opportunity to 
clearly identify different frequency regions [5]. This can be observed in Figure 1. The targeted resonance is highlighted as region $\mathrm{C}$ and will be referred to as the resonant pole region. It had been suggested by James et al [5] that this resonance is due to residual inductance. The current paper describes an approach of targeting the distributed interaction of the residual inductance and the winding capacitance.
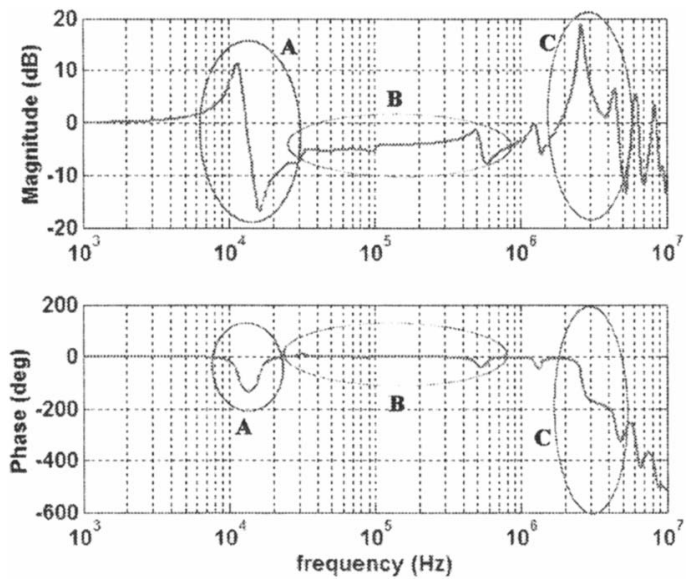

Figure 1. Frequency Response from Bushing to Neutral Region A - Self Resonant Frequency Region B - Capacitive Region Region C-Resonant Pole Region

Figure 2 depicts two disc pairs (DP) of the equivalent circuit of an interleaved winding. Beyond the self resonant frequency (Region A in Figure 1), the frequency characteristic of the winding is dominated by the capacitance between the interleaved turns in each disc and the capacitance to ground ( $\alpha$ ratio), as per Region B in Figure 1. However, it is suggested that at higher frequencies $(>1 \mathrm{MHz})$, residual inductive paths, which have not been effectively removed via capacitive coupling between turns, will interact with the capacitive elements of the winding to generate high frequency resonances (Region $\mathrm{C}$ in Figure 1). Since these inductive elements are consistent with the layout and construction of the winding, their symmetry can be exploited for PD location purposes.

In the work by Pedersen [14], Zambardino argued that for an extremely short transient burst (several nanoseconds), the series capacitance would be determined by the first few turns of the interleaved disc, and the remaining turns would be in series with each other (including the shunt capacitance to ground so a resulting change in the alpha ratio). This argument in conjunction with the premise of parasitic/residual inductances, reduces the equivalent circuit to that observed in Figure 3. If an upper frequency bound of $10 \mathrm{MHz}$ is assumed then according to the traveling wave criterion no lumped parameter can be greater than a few meters which will be satisfied in most instances if each disc pair is modeled as per Figure 3.

\section{B. PD Location}

The fundamental premise behind the proposed PD location technique is that a partial discharge can be considered to approximate a current impulse [4]. The current at the neutral will provide the impulse response and hence, in the frequency

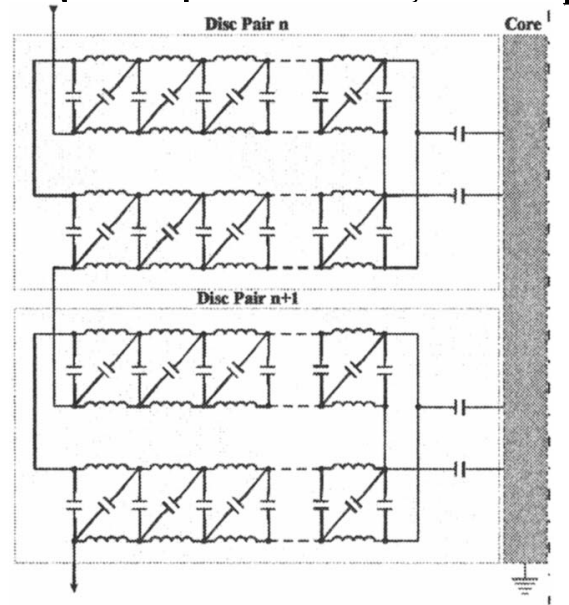

Figure 2. Interleaved Winding Equivalent Circuit for two sections
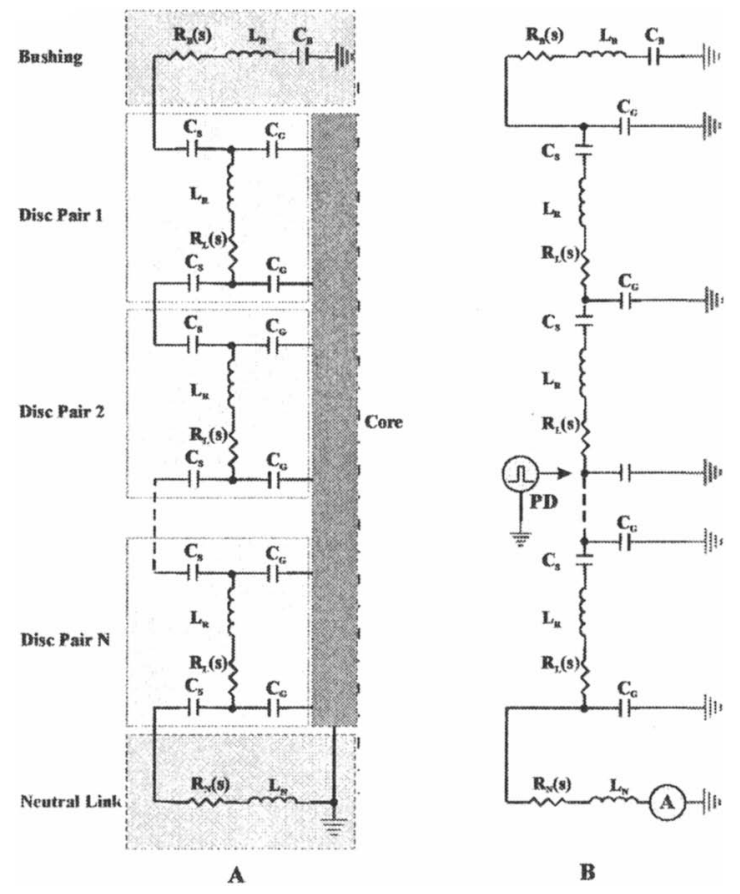

Figure 3. High Frequency Distributed Model

A: High Frequency Model, B: Simplified High Frequency Model $\mathrm{v}_{\mathrm{G}}=$ Shunt Capacitor $(\mathrm{V}), \mathrm{v}_{\mathrm{S}}=$ Series Capacitor $(\mathrm{V}), \mathrm{i}_{\mathrm{L}}=$ Inductor $(A)$

domain, the transfer function from the PD site to the neutral. Since the poles of the transfer function are system dependent they will remain constant, whilst the zeros will be indicative of the injection point. A similar approach at lower frequencies $(<500 \mathrm{kHz})$ using the standard ladder network has been discussed in various papers $[4,6,7]$. The state space representation of the circuit shown in Figure 3B is:

$$
\begin{aligned}
& \dot{x}=A x(t)+B u(t) \\
& y=C x(t)
\end{aligned}
$$

Where 


$$
\begin{aligned}
& x=\left[\begin{array}{lllllllll}
v_{G 1}(t) & \cdots & v_{G N}(t) & v_{S 1}(t) & \cdots & v_{S v}(t) & i_{L 1}(t) & \cdots & i_{L N}(t)
\end{array}\right]^{T} \\
& A=\left[\begin{array}{lll}
A_{G G} & A_{G S} & A_{G L} \\
A_{S G} & A_{S S} & A_{S L} \\
A_{L G} & A_{L S} & A_{L L}
\end{array}\right], A_{G G}=A_{G S}=A_{S G}=A_{S S}=0 \\
& A_{G L}=\left[\begin{array}{cccc}
\frac{-1}{C_{B}} & 0 & \cdots & 0 \\
\frac{1}{C_{G}} & \frac{-1}{C_{G}} & 0 & \ddots \\
0 & \ddots & \ddots & \ddots \\
\vdots & \ddots & \frac{1}{C_{G}} & \frac{-1}{C_{G}}
\end{array}\right], A_{S L}=\left[\begin{array}{cccc}
\frac{1}{C_{s}} & 0 & \cdots & 0 \\
0 & \frac{1}{C_{s}} & \ddots & \vdots \\
\vdots & \ddots & \ddots & 0 \\
0 & \cdots & 0 & \frac{1}{C_{s}}
\end{array}\right] \\
& A_{L G}=\left[\begin{array}{ccccc}
\frac{1}{L_{B}} & \frac{-1}{L_{B}} & 0 & \cdots & 0 \\
0 & \frac{1}{L_{R}} & \frac{-1}{L_{R}} & \ddots & \vdots \\
\vdots & \ddots & \ddots & \ddots & 0 \\
\vdots & \ddots & 0 & \frac{1}{L_{R}} & \frac{-1}{L_{R}} \\
0 & \cdots & \cdots & 0 & \frac{1}{L_{N}}
\end{array}\right] A_{L S}=\left[\begin{array}{cccc}
\frac{-1}{L_{B}} & 0 & \cdots & 0 \\
0 & \frac{-1}{L_{R}} & \ddots & \ddots \\
\vdots & \ddots & \ddots & 0 \\
0 & \ddots & 0 & \frac{-1}{L_{N}}
\end{array}\right], \\
& A_{L}=\left[\begin{array}{cccc}
\frac{-R_{B}}{L_{B}} & 0 & \cdots & 0 \\
0 & \frac{-R_{L}}{L_{R}} & \ddots & \ddots \\
\vdots & \ddots & \ddots & 0 \\
0 & \ddots & 0 & \frac{-R_{N}}{L_{N}}
\end{array}\right]
\end{aligned}
$$$$
B=\left[\begin{array}{llllllllll}
0 & \cdots & 0 & \frac{1}{C_{G k}} & 0 & \cdots & 0_{(N)} & \cdots & 0_{(3 N)}
\end{array}\right]^{T} \text { and } C=\left[\begin{array}{llll}
0 & \cdots & 0 & 1_{(3 N)}
\end{array}\right]
$$

By taking the Laplace Transform of Equation (1), the transfer function from the partial discharge source node to the neutral point is given by:

$$
H(s)=\frac{Y(s)}{U(s)}=C(s I-A)^{-1} B
$$

Note that a partial discharge can be considered to be a current impulse [4], hence the input $U(s) \approx 1$.

Matrix B locates the PD injection node $\mathrm{k}$, of $\mathrm{N}$ nodes. The poles of the transfer function are system dependent and will remain constant irrespective of the PD injection location [7]. The system zeros, however, will shift with the PD site and it is this feature that is often exploited for partial discharge location [7].

$R(s)$ represents the frequency dependent loss terms due to dielectric loss, proximity and eddy current effects. The loss elements directly impact on the levels of resonant damping that are observed.

\section{EXPERIMENTAL RESUlts}

A single $66 \mathrm{kV} / 25 \mathrm{MVA}$ interleaved transformer winding with the secondary and iron core removed was used for testing purposes. The core was replaced with a split aluminum cylinder to maintain shunt capacitive relationships with respect to the system frequency response. The winding consisted of 19 disc pairs with 80 turns per pair. A 73kV Micafil Bushing terminated one end of the winding with the other terminated at ground. The bushing is modeled as $100 \mathrm{pF}$ capacitance.

\section{A. Analysis of the Frequency Response}

Based on the assumption that a transformer winding can be viewed as a reciprocal network, the injection of a voltage at the neutral and recorded at the bushing will be the same as a current injected at the bushing and recorded at the neutral [4]. By application of the theory of reciprocity one is able to use a vector analyzer (VA) with a termination resistance of $1 \mathrm{M} \Omega$ to record the frequency response of the transformer under test. The recorded response will be equivalent to an ideal current impulse injected at the bushing and hence provides an insight into the frequency domain of a recorded PD signal (Figure 1).

The proposed high frequency model was applied to data captured by the vector analyzer within Region $\mathrm{C}$, with the aim of matching the two resonant pole frequencies (Figure 4). As above, the poles are system dependent and will not change with PD location and hence obtaining a satisfactory fit is essential for testing the applicability of the model.
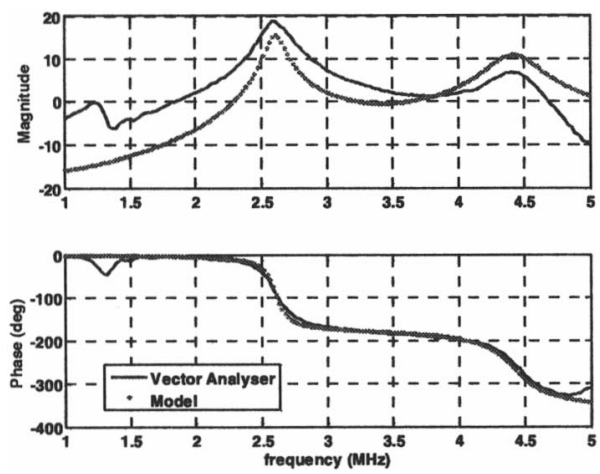

Figure 4. Bushing to Neutral Frequency Response in Region C

\section{B. "Live" PD Injection}

The "live" discharge source was an oil-immersed needle-plate electrode with a pressboard sandwiched in between. At the applied voltage of $7.5 \mathrm{kV}$, steady PDs of $\sim 100 \mathrm{pC}$ were generated and injected into the winding at various test locations. The measurement equipment consisted of a DC$100 \mathrm{MHz}$ broadband current probe/amplifier terminated into a 
digital oscilloscope. Figure 5 depicts the PD injection experimental setup and Figure 6 presents the digitally filtered time response of a PD injected into disc pair 15 and recorded at the neutral.

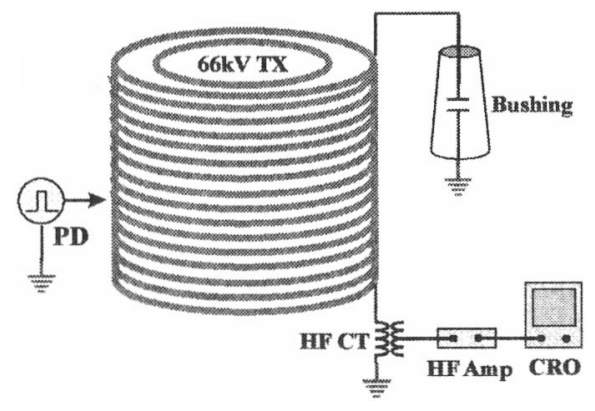

Figure 5. Test Configuration

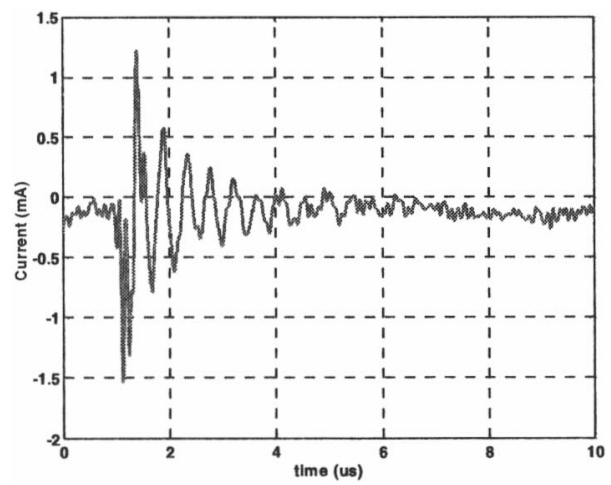

Figure 6. PD Time Response at Neutral Injection point at disc pair 15 of 19 with $2 \mathrm{~ns}$ sampling interval

\section{Testing and Measurement Influence}

Several papers have discussed the need to account for the influence of the test and measurement equipment of frequencies greater than a few hundred $\mathrm{kHz}$. To determine the level of influence exhibited by the testing equipment, a partial discharge signal was recorded at the point of entry into the winding and compared with the neutral terminated measurements. Using a fast Fourier transform (FFT), the magnitude of the frequency responses were compared (Figure 7). As can be observed, the area of interest $(1-10 \mathrm{MHz})$ for the input signal has a relatively flat spectral response. This correlates nicely with the initial assumption that the PD behaves as a near perfect impulse i.e. possesses a flat spectrum over all frequencies, or at least in this case, the frequencies of interest.

Another point to note is the resonant peak observed at $18 \mathrm{MHz}$. This resonance is most likely due to the resonance interaction of the coaxial injection cable.

\section{Analysis Procedure for PD location with HF Model}

Live partial discharges were applied to various locations in the winding. To estimate the PD site of origin using the proposed model, the following steps were followed:-

- Inject a PD and record the samples at the neutral

- Apply an FFT to obtain the frequency response
- Estimate the resonant pole locations $\left(P_{1}, P_{2}\right)$

- Estimate the first zero $\left(Z_{1}\right)$

- Adjust the model parameters to match pole locations

- Adjust the model "PD injection" point to match $Z_{1}$

Figure 8 shows a comparison at various locations in the winding between the PD frequency response and the predicted response. Tables I and II highlight the zero variation and the corresponding estimation errors. By comparing the data and model's first zero $\left(Z_{1}\right)$ after the dominant resonant pole $\left(P_{1}\right)$, an approximate PD location is achieved. However, the prediction method has estimation errors of up to $21 \%$ near the ends of the winding. The estimation errors tend to reduce as the injection point moves towards the middle section of the winding. This is highlighted in Figure 9. Further improvement can be obtained at the bushing end by making the assumption that the absence of a zero between $P_{1}$ and $P_{2}$ is indicative of a resonant pole response and hence PD injection at Disc Pair 1 (Table I and II).

As an aside it is worth noting that the assumption of reciprocity is justified by comparing Figure 1 Region $\mathrm{C}$ with Figure 8a.

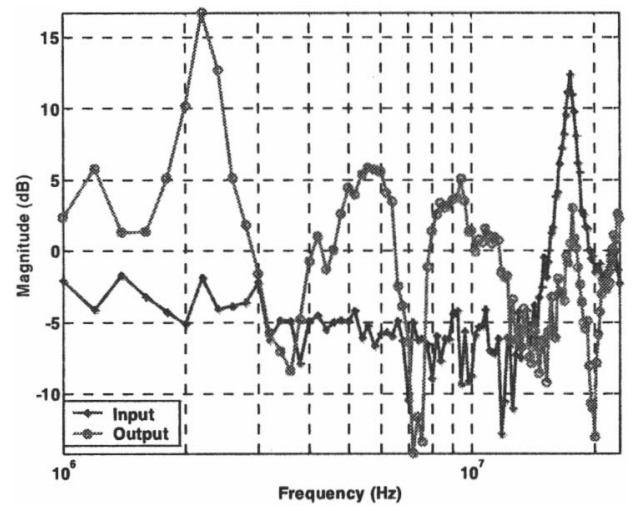

Figure 7. PD Input versus Output (Site of Origin DP3)

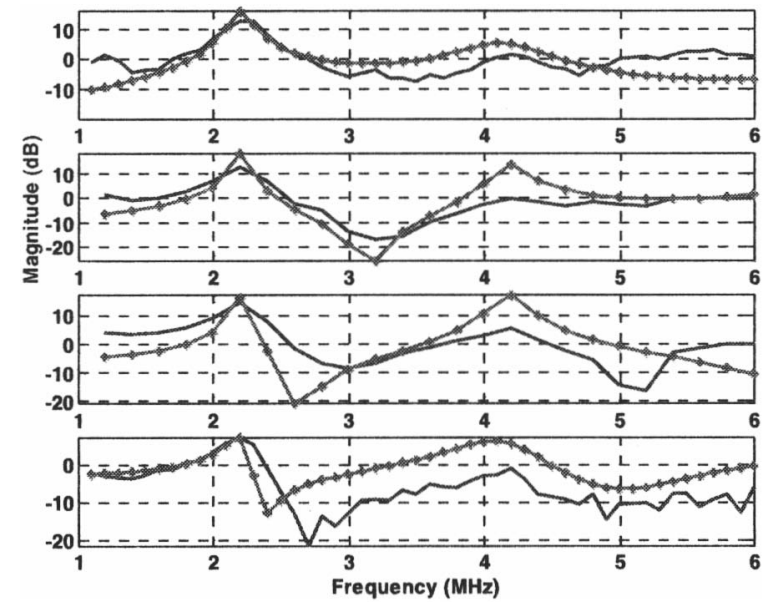

Figure 8. PD Frequency Response versus model at various injection points

(a): Data/Model Disc Pair 1/19

(b): Data/Model Disc Pair 9/19

(c): Data/Model Disc Pair 13/19

(d): Data/Model Disc Pair 17/19 


\section{E. Analytical Approach}

By observation, an analytical approach can be applied that improves on the parameter model estimates. The zero $Z_{1}$ will shift from $P_{2}$ to $P_{1}$ with a corresponding shift in the injection location towards the neutral. Mathematically this can be expressed as:-

\%Winding $=1-\left(\frac{\mathrm{P}_{2}-\mathrm{Z}_{1}}{\mathrm{P}_{2}-\mathrm{P}_{1}}\right)$ where $P_{1} \leq Z_{1} \leq P_{2}$

The results are also included in Tables I and II and Figure 9

TABLE I

ESTIMATED $Z_{1}$ FREQUENCY FOR DATA, MODEL \& ANALYTICAL APPROACHES

\begin{tabular}{|c|ccc|}
\hline $\begin{array}{c}\text { Disc Pair } \\
1 / 19\end{array}$ & $\begin{array}{c}\text { Data }(\mathbf{M H z}) \\
\text { Model }(\mathbf{M H z})\end{array}$ & $\begin{array}{c}\text { Analytical } \\
\text { (MHz) }\end{array}$ \\
\hline $5 / 19$ & 4.6 & *** & 4.1 \\
\hline $5 / 19$ & 3.9 & 5.8 & 3.9 \\
$7 / 19$ & 3.8 & 4.4 & 3.7 \\
\hline $9 / 19$ & 3.2 & 3.6 & 3.5 \\
\hline $11 / 19$ & 3.2 & 3.2 & 3.3 \\
\hline $13 / 19$ & 3.0 & 2.8 & 3.1 \\
\hline $15 / 19$ & 3.0 & 2.7 & 2.9 \\
\hline $17 / 19$ & 2.8 & 2.5 & 2.7 \\
\hline *** Pure resonant pole response (no zeros) & 2.4 & 2.5 \\
\hline
\end{tabular}

TABLE II

ESTIMATION ERROR (\% WINDING)

\begin{tabular}{|c|c|c|}
\hline Disc Pair & Model & Analytical \\
\hline $1 / 19$ & $+16 \%(* * * 0 \%)$ & $0 \%$ \\
\hline $3 / 19$ & $+16 \%$ & $0 \%$ \\
\hline $5 / 19$ & $+11 \%$ & $-5 \%$ \\
\hline $7 / 19$ & $+5 \%$ & $+11 \%$ \\
\hline $9 / 19$ & $0 \%$ & $0 \%$ \\
\hline $11 / 19$ & $-5 \%$ & $0 \%$ \\
\hline $13 / 19$ & $-16 \%$ & $-11 \%$ \\
\hline $15 / 19$ & $-21 \%$ & $-11 \%$ \\
\hline $17 / 19$ & $-21 \%$ & $-21 \%$ \\
\hline
\end{tabular}

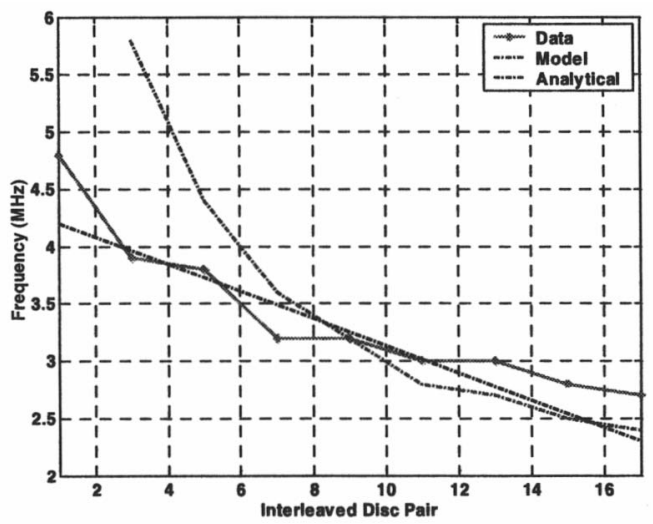

Figure 9. Frequency of $Z_{1}$ for Data, Frequency and Analytical approach

\section{CONCLUSION}

This paper proposes a narrowband high frequency model that can be utilized for partial discharge location of an interleaved transformer winding. This is achieved by targeting a frequency band around a residual inductance induced high frequency resonance. The accuracy achieved allows the PD location to be determined to within twenty percent of the winding. This technique has several advantages. The algorithm does not require an in depth material or structural knowledge base and it does not require calibration. It alternatively leverages on the three degrees of freedom offered by parameters $\mathrm{Cs}, \mathrm{Cg}$ and $\mathrm{Lr}$ to match the targeted resonant poles of a detected partial discharge frequency response. The algorithm also takes advantage of the high frequency nature of partial discharges by working at relatively high frequencies $(>1 \mathrm{MHz})$, which provides a higher degree of sensitivity $(<100 \mathrm{pC})$. And finally, measurements are taken at one point only.

\section{ACKNOWLEDGMENT}

The authors would like to thank the University of New South Wales, Australia, for access to their testing facilities.

\section{REFERENCES}

[1] J. P. Steiner and W. L. Weeks, "A new method for locating partial discharges in transformers," 1990, pp. 275-281

[2] Y. Zhou, A. I. Gardiner, G. A. Mathieson, and Y. Qin, "New methods of partial discharge measurement for the assessment and monitoring of insulation in large machines," 1997, pp. 111-114.

[3] Z. D. Wang, P. A. Crossley, and K. J. Cornick, "A simulation model for propagation of partial discharge pulses in transformers," 1998, pp. 151155 vol.1.

[4] S. Xu, S. D. Mitchell, and R. H. Middleton, "Partial Discharge Localization for a Transformer Based on Frequency Spectrum Analysis," in AUPEC Christchurch New Zealand, 2003.

[5] R. E. James, B. T. Phung, and Q. Su, "Application of digital filtering techniques to the determination of partial discharge location in transformers," Electrical Insulation, IEEE Transactions on [see also Dielectrics and Electrical Insulation, IEEE Transactions on], vol. 24, pp. $657-668,1989$.

[6] Z. D. Wang, S. N. Hettiwatte, and P. A. Crossley, "A measurementsbased discharge location algorithm for plain disc winding power transformers," Dielectrics and Electrical Insulation, IEEE Transactions on [see also Electrical Insulation, IEEE Transactions on], vol. 12, pp. 416-422, 2005.

[7] S. N. Hettiwatte, P. A. Crossley, Z. D. Wang, A. Darwin, and G. Edwards, "Simulation of a transformer winding for partial discharge propagation studies," 2002, pp. 1394-1399 vol.2.

[8] R. Ludwig and P. Bretchko, RF Circuit Design Theory and Applications: Prentice Hall, 2000.

[9] G. B. Gharehpetian, H. Mohseni, and K. Moller, "Hybrid modelling of inhomogeneous transformer winding for very fast transient overvoltage studies," Power Delivery, IEEE Transactions on, vol. 13, pp. 157-163, 1998.

[10]W. J. McNutt, T. J. Blalock, and R. A. Hinton, "Response of Transformer Windings to System Transient Voltages," IEEE Transactions on Power Apparatus and Systems, vol. PAS-93, pp. 457-467, 1974.

[11]W. W. L. Keerthipala and P. G. McLaren, "A multiconductor transmission line model for surge propagation studies in large a.c. machine windings," 1990, pp. 629-632 vol.2.

[12]Y. Shibuya, S. Fujita, and E. Tamaki, "Analysis of very fast transients in transformers," Generation, Transmission and Distribution, IEE Proceedings-, vol. 148, pp. 377-383, 2001.

[13]A. Akbari, P. Werle, H. Borsi, and E. Gockenbach, "A continuous parameter high frequency model based on travelling waves for transformer diagnostic purposes," 2002, pp. 154-157.

[14]A. Pedersen, "On the Response of Interleaved Transformer Windings to Surge Voltages," IEEE Transactions on Power Apparatus and Systems, vol. 82, pp. 349-356, 1963 